\title{
Novel Methods to Drive Pattern Engineering Through and For Enhanced Use of 3D Technologies
}

\author{
Emma SCOTT ${ }^{1}$, Simeon GILL², Carol MCDONALD ${ }^{3}$ \\ ${ }^{1}$ Fashion Should Empower, Vancouver Island BC, Canada; \\ 2 The University of Manchester, Manchester, UK; \\ ${ }^{3}$ Gneiss Concept, Washougal, WA, USA \\ https://doi.org/10.15221/19.211
}

\begin{abstract}
Key obstacles to apparel digitization, frequently and symptomatically observed as limitations within 3D technologies, can be summarized as a lack of theory to quantify body shape and garment ease. Whether discussing garment fit on parametric avatars, automated virtual garment design, mass garment customization, improved garment sizing, or accurate size prediction, apparel digitization has repeatedly been hindered by a lack of theory linking the garment and its shaping requirements to the human form. This is because neither 1D (tape measure) dimensions nor a 3D flattened polygon mesh can quantify body shape with respect to developing garment patterns conducive to apparel manufacturing.

While novel pattern-engineering theory offers body-to-pattern guidance to isolate ease and body shape dimensions, acquiring the required measurements (outside the scope of both ISO boundaries and current measurement extraction software) has proven prohibitive. To prove scan-to-pattern digital viability a study explored the potential for updated measurement extraction software to access underutilized body scan data to drive Clone Block $B$ body-to-pattern theory. Solidworks 3D software was used to manually extract measurements from a body scan and Intelligent Shaping ${ }^{\circledR}$ software was used for automated Clone Block $®$ pattern development. The suitability of the measurements for supporting body-to-pattern theory was then tested based on the ability of the resulting pattern to hug body morphology while maintaining horizontal and vertical fabric grain.

The results of the study indicate automated measurement acquisition to drive Clone Block® shaping methodologies for scan-to-pattern automation is feasible. Based on the premise that garment fit is the result of fit-reality (body shape) plus fit-preference (ease), this study is significant in offering a means by which underutilized scanned body data may be accessed to quantify body shape and ease. Further study should confirm that updated measurement extraction software can automate the retrieval of measurement data and the Clone Block $®$ scan-to-pattern methods (used extensively in a bespoke environment) work on an extended range of body shapes including bodies with dysmorphia. Discussions surrounding ease distribution for body shape should also be studied for foundational mathematical fit-preference and ease theory.
\end{abstract}

Keywords: Clone Block, body shape parameterization, mass garment customization, virtual garment design

\section{Introduction}

Traditional pattern-drafting methods focus first on drafting a basic garment shape and secondly on garment fit. This allows a first stage pattern to be created from a limited set of body measurements with the understanding that this initial pattern will be corrected following sampling and try-on of a test garment. [1] Garment sampling is a costly process for the apparel industry and it is here that 3D technologies have had the greatest impact by allowing test garments to be virtually sewn and fit, saving both person-hours and materials in the process. [2] While this has advanced apparel digitization, it has also highlighted inherent flaws within pattern-engineering theory. The need to correct garment fit post pattern construction speaks to a lack of direct relationship between anthropometry and pattern. Inherent flaws within real-world traditional pattern-engineering theory have merely been transferred to digitalworlds.

When we look at the capabilities of technology to make illusions appear very life-like and real, it is easy to assume 3D technologies have solved the problems of real-world garment fit. [3] [4] Translating the $3 \mathrm{D}$ garment (which is essentially a polygon mesh in the virtual environment) from a $3 \mathrm{D}$ avatar to a developable pattern for manufacturing, however, has had limited success. First of all, 3D garments are not bound by the constraints of real-world fabric grain. While 3D printed garments have experimented with bringing the polygon mesh to the real-world, apparel manufacturing remains firmly rooted in traditional fabrics defined by the properties of weft and warp threads. [5] It must therefore be acknowledged that while current technologies CAN make any pattern fit any body shape within the 3D 
realm, [6] these patterns do not always translate well to the physical world. As witnessed by difficulties with traditional MTM (made-to-measure) garments accommodating outlier figures, translating virtual garments to real-world garments bound by the constraints of body morphology and fabric grain remain unperfected. [5] Regardless of the realism displayed on a monitor, the problems with real-world garment fit remain unchanged by the technologies designed to improve them. [2]

The need for a direct relationship between anthropometry and garment pattern has become critical. Apparel digitization depends upon a clear pattern-to-person relationship and customer insistence on body shape inclusive-sizing necessitates it. The key obstacles can be summarized as a lack of theory to quantify body shape and garment ease. [1] [7] [8] [9] While novel software to address these concerns exists, the extensive and detailed body data required is not within the scope of current measurement extraction software, nor completely defined within traditional ISO standards. The study discussed herein, will demonstrate the viability of automating novel measurement practices to support novel patternengineering for body shape theory (Clone Block®), and thereby establish a direct body-to-pattern relationship. Positive domino effects on apparel digitization are also discussed.

\title{
2. Introduction
}

Fit-reality dimensions (2D origami representation of the body) are substantially different from 1D (tape measure) dimensions and this little observed fact is the fatal flaw at the root of garment fit woes and stalled apparel digitization. As illustrated in Figure 3, garment fit must account not only for 1D body dimensions but also the 2D origami grey zones required to shape pattern to body. [7]

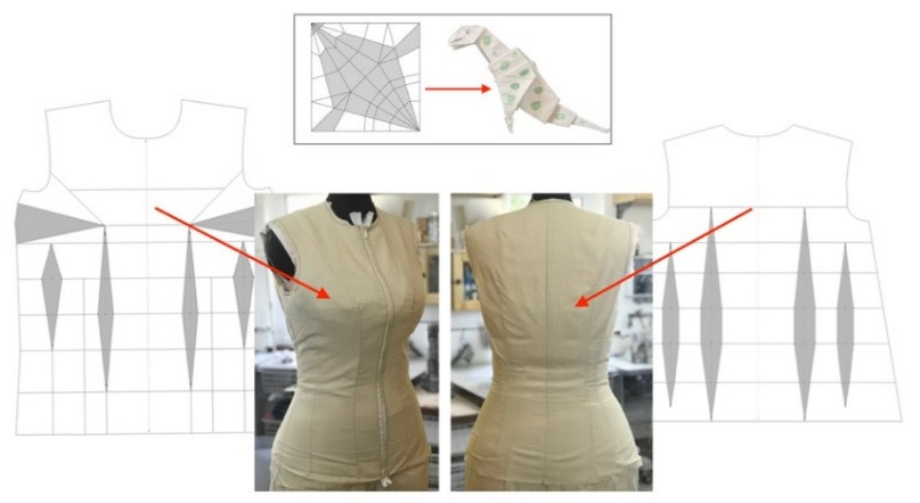

\begin{abstract}
To manipulate a $2 \mathrm{D}$ pattern to a 3D shape areas must be folded out and hidden. With regard to garment fit, these hidden grey zone areas either shape pattern to body, become factors for calculating ease distribution, or contribute to hidden ease effecting fit preference calculations.
\end{abstract}

Figure $1 \sim$ The relationship of origami to pattern-engineering for body shape.

Discussions surrounding garment fit are greatly complicated by subjective views regarding aesthetics. What one person deems good fit another may consider poor fit, thereby negating any possibility of attaching a set value to fit. Indeed, the very nature of design is to break constraints and/or rules such that artistic expression may push boundaries to inspire. How to quantify fit without value constraints has been a dilemma for pattern-engineers that inevitably has become problematic for apparel digitization. To solve this dilemma there must a ground zero, a baseline against which fit can be gauged. The natural, and incorrect, assumption for this baseline has been 1D body dimensions (tape measure). The problem with this oversimplification is, as illustrated in Figure 2, two individuals with the same 1D dimensions can have very different shapes, rendering current methods of comparing 1D body dimensions against fit model or size chart dimensions ineffective and fit preference calculations incorrect.
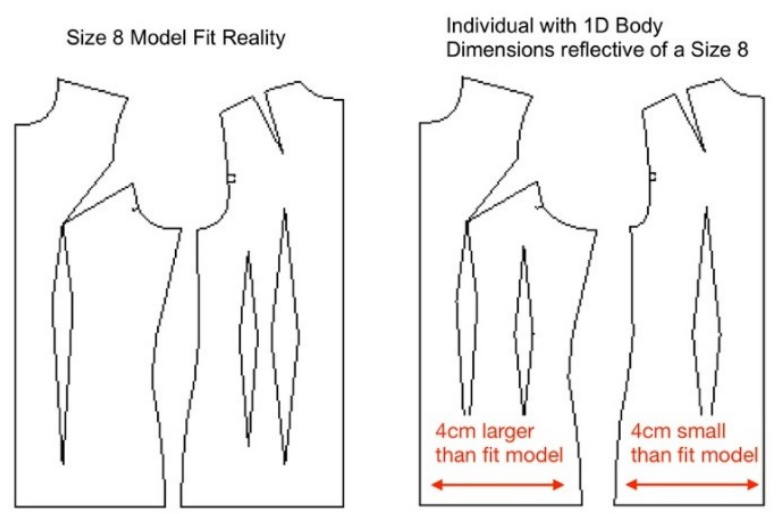

\author{
A critical garment fit assessment, considering the \\ origami pattern requirements for body shape reveals \\ quantifiable fitting data not revealed with 1D \\ dimensions and essential for machine learning \\ algorithms. i.e. \\ 1. Front hip is $4 \mathrm{~cm}$ small but back girth is sufficient to \\ accommodate body. \\ 2. Dresses will pull tight over abdomen but will be \\ loose at back waist. \\ 3. Pants will pull tight over abdomen. Choose fabrics \\ with high fit tolerance.
}

Figure $2 \sim 1 D$ versus $2 D$ body measurements. 
1D analysis permits an assessment of body widths and lengths, 3D assessment provides aesthetic clues as to body shape, but only the 2D origami pattern identifies the interplay between body shape and fabric grain that is viewed as garment fit. Only the 2D pattern, representing the garment fabric, precisely pinpoints darting and shaping to identifying how fabric and ease are distributed around the body. [8] As illustrated in Figure 3 only through 2D body shape critique can any significant analysis with regard to garment fit be undertaken.

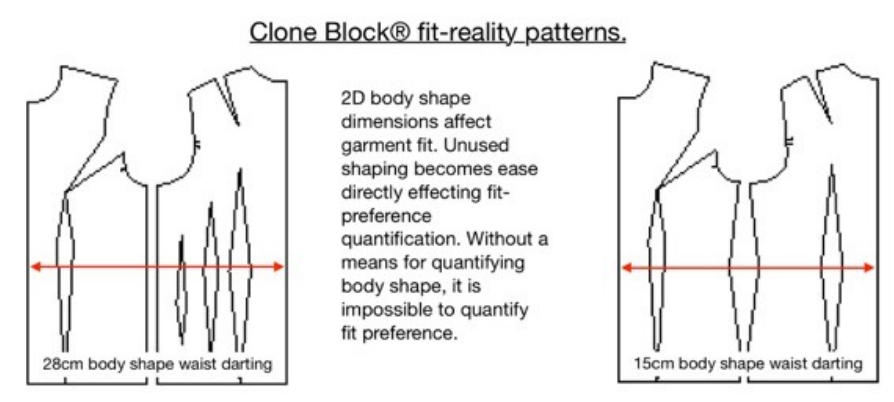

Block adapted for pattern with ease and darting removed.

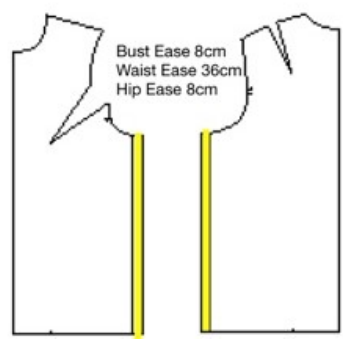

At first glance it appears these patterns have had the same amount of ease added but this is an incorrect assumption negatively affecting fit preference calculations. $2 \mathrm{~cm}$ to each side seam equals of $8 \mathrm{~cm}$ of ease but this does not account for the hidden ease due to body shaping.

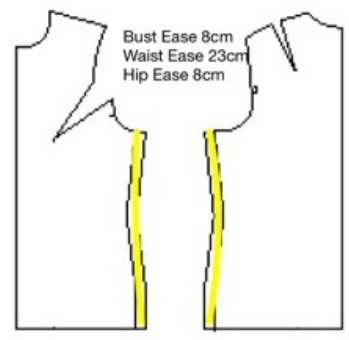

Figure $3 \sim$ Hidden ease affecting fit preference calculations.

There are many texts describing effective methods of pattern-drafting to achieve a variety of pattern shapes. None adequately support what will be referred to as pattern-engineering. Consequently, each recommends methods believed to be useful for shaping pattern to body in post-drafting garment fittings. [10] [11] [12] Furthermore, traditional pattern-drafting theory is inclusive of ease, making it difficult to isolate the pattern dimensions specifically required for body shape from that required for ease alone. [13] Pattern blocks are the base patterns from which all garments originate. It is through manipulation of these blocks that garment patterns are derived. Therefore, a body-shaping error in the parent pattern block will be inherent in any derived child patterns. While it can at first appear implausible the apparel industry evolved without a clear methodology for quantifying body shape it must be understood that patterns without ease are difficult if not impossible for a wearer to put on. The inclusion of ease in the engineering of traditional pattern blocks is partially to permit body movement sufficient for dressing and partly to account for body-shape. Prior to 3D environments for virtual testing of fit, physical fit-testing of patterns without ease would have been difficult if not impossible without the inevitable garment sampling. Further, without computers the myriad of measurements and calculations required to assess body morphology were simply impractical for the fast-paced environment of off-the-rack apparel design.

3D technology has undoubtedly provided the optimal environment for exploring body-to-pattern relationships. Attempts at streamlining this process, however, have had limited success. [2] Initially, difficulties arose from a lack of accuracy with regard to avatar and fabric properties. While the realism of $3 \mathrm{D}$ environments continues to improve, methods of flattening an avatar into a polygon shape to explore body-to-pattern relationships have proven problematic in that the theory is not easily relatable to established apparel manufacturing practices. [14] Research to address this continues. [15]

Some researchers have suggested the need for more body measurement data to relate to pattern theory. [16] A widespread belief that technology should minimize user inputs rather than increase them has, for the time being nulled such suggestions. Other research has explored side seam placement and customized darting as ways to assess body shape, where body shape is predominantly affected by front-to-back variations. [17] However, the results of such research are infrequently applied in practice. Intelligent Shaping ${ }^{\circledR}$ software, using the Clone Block ${ }^{\circledR}$ method of pattern-engineering, leads us to support a direct body-to-2D pattern relationship. However, measurement acquisition has not been fully developed. While the software has successfully been used in a bespoke garment environment, the extensive body data required has proven problematic. Depending on the ability of a subject to remain standing for an extended period, the measuring process requires sixty to ninety minutes. There are also difficulties keeping landmarking planes aligned for the duration of the process. Due to the natural 
curvature of the body it is very common for the horizontal landmark strings to move during measurement, making hand data acquisition neither practical nor completely accurate. While the task is best suited for automated measurement extraction from point cloud data, current extraction software, developed according to ISO standards and traditional pattern-drafting theory, accesses only a small portion of the required data. This is largely due to historic constraints of manual measurements setting the agenda for what is required to draft a pattern.

This paper will highlight how underutilized data from 3D body scans combined with novel patternengineering theory provide foundational logic to address flaws within traditional pattern-engineering theory. Such theory lays the foundation to further propel apparel digitization into a world where garment sketches are simultaneously produced in 3D and can be ready for manufacturing within seconds.

\section{Method}

For the study a single individual was both hand and virtually measured. Since Clone Block® theory was developed to work within traditional apparel manufacturing practices, the main difference from traditional measuring protocols is the segmentation of full body dimensions for assessing body weight and height distribution. Consequently, while it was understood that current body scanned measurement data available would be insufficient, manually extracting the data using current software was considered plausible. The complexities of placing multiple sagittal and coronal planes was underestimated and current apparel measurement extraction software was found insufficient for this study. Size Stream studio software provided the most correlated automated landmark slices but, without the ability to reference the required intersecting tilted sagittal and coronal planes, manual segmentation of the transverse slices for relative circumference division would have amounted to guessing. The same complication with landmarking was encountered in 3D Measure Up software. Had it been possible to have multiple sagittal and coronal planes intersect the slices (for accurate segmentation of full circumferences) landmarking and manual extraction of the required measurements could have been accomplished with either software. Therefore, to prove automated digital viability of measurement extraction suitable for Clone Block $B$ theory, an experiment using Solidworks software to manually extract measurements was undertaken.

All landmarks are positioned according to caliper widths and depths making the methodology well suited for automation. For the torso, the key landmarks are the highbust and gluteal fold. All other torso landmarks are proportionally derived from these key landmarks. For the legs, the key divisions below the gluteal are knee and highest ankle with all other leg divisions derived proportionally between these landmarks.

Intelligent Shaping ${ }^{\circledR}$ software was then used to create base pattern blocks using the Clone Block® method of pattern-drafting. Patterns were made from both the digitally acquired and hand acquired measurements. Garment fit was assessed based on the ability of the pattern block to match body morphology while maintaining horizontal and vertical fabric grain.

\subsection{Measurement Data Acquisition}

\subsubsection{Hand Measuring}

Figure 4 illustrates the landmarking procedure for hand measurement. It is imperative the subject is measured in minimal clothing and if applicable, with breasts separated in an encapsulated bra and supported above the inframammary fold.

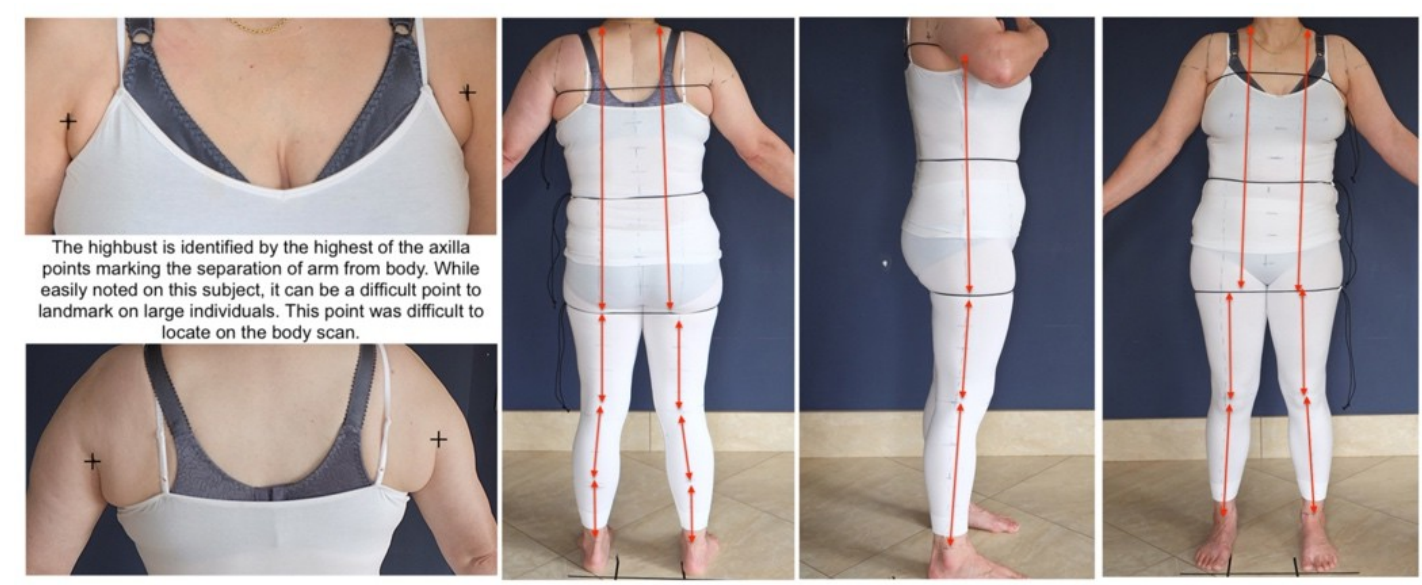

Figure 4 Hand landmarking and measuring for Clone Block® theory. 


\subsubsection{Digital Measuring}

For digital measuring a $3 \mathrm{MB}$ ply file from a Size Stream body scanner was used. First the file was converted to a stl file in Meshlab. Then it was repaired in Netfabb and further optimized to reduce $20 \mathrm{~K}$ faces to $5 \mathrm{~K}$ faces again in Meshlab. The final file was $4 \mathrm{~KB}$ was then imported into Solidworks. The use of both planar and tilted sagittal, coronal, and transverse planes was used to successfully segment full body dimensions for effective analysis of body weight and height distribution. Solidworks sketching and measuring functions were then used to extract body contour measurements between intersecting planes. The process, illustrated in Figure 5, was tedious and more time consuming than measurements acquired by hand but with all 400 plus measurements derived from intersecting planes, placed according to caliper widths and depths, the inclusion of the technique in current measurement extraction software appears highly plausible.

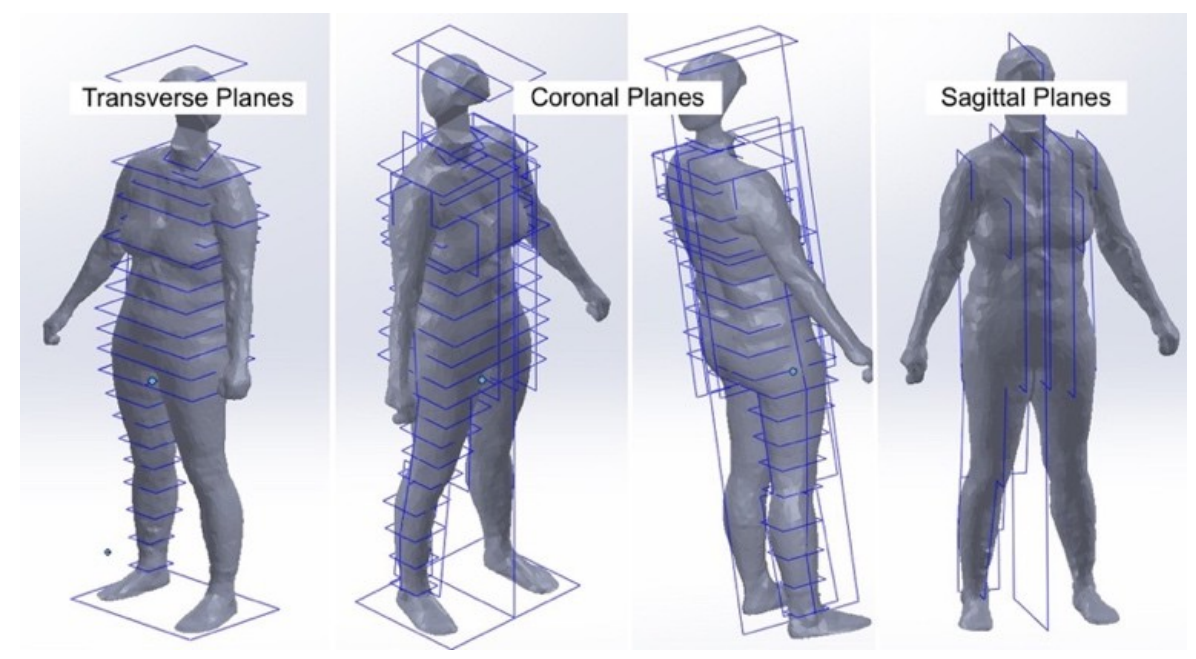

Figure 5 Digital landmarking and measuring for Clone Block® theory.

\subsection{Testing Effectiveness of Measurements for Body-to-Pattern Relationship}

Intelligent Shaping ${ }^{\circledR}$ demonstration software was used to create a garment pattern without ease. The software uses the Clone Block $®$ method of pattern-engineering and uses over 400 body dimensions to parameterize a jumpsuit block reflective of body morphology. The resulting .dxf pattern was then exported to PAD CAD software for printing and sampling. Patterns for both digital and physical measurements were generated. Since the patterns were constructed mainly exclusive of ease, garment fit was assessed based on the ability of the pattern to mold to body morphology while maintaining fabric grain in both the horizontal and vertical axis. Minor ease $(.75 \mathrm{~cm})$ was added to torso side seams $(3 \mathrm{~cm}$ total) so as not to restrict breathing much. Figure 6 illustrates the patterns derived from both hand and digital measuring.

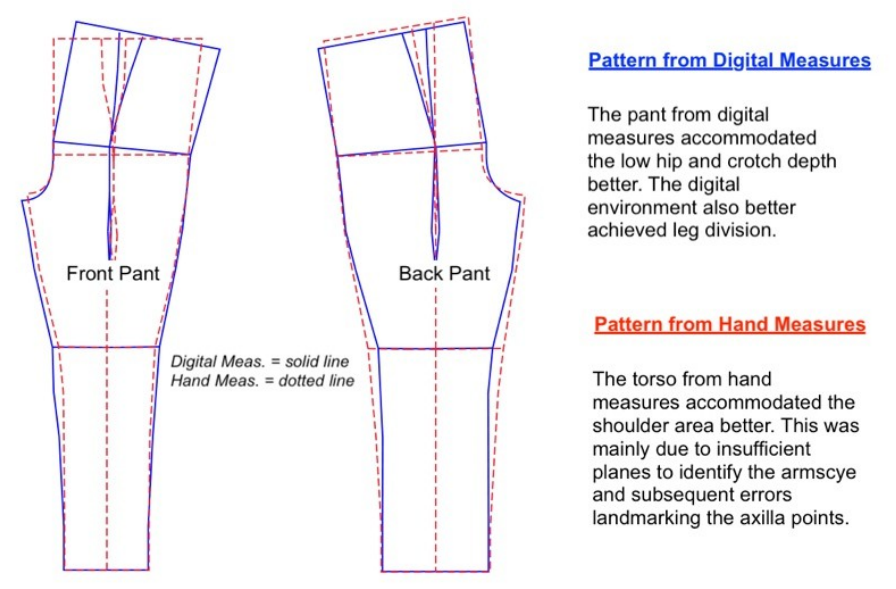

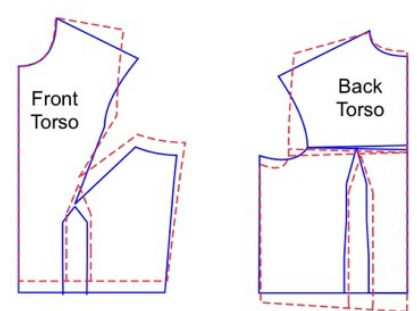

Digital Meas. $=$ solid line

Figure 6 - Patterns from digital versus hand anthropometry. 


\section{Results}

This study demonstrates a way in which underutilized scanned body data may be accessed to support theory with a direct body-to-pattern relationship. The use of both planar and tilted sagittal, coronal and transverse planes successfully segmented full-body girth circumferences and lengths into the segmented body girths as required for Clone Block® theory. While the resulting pattern block offers a fit similar to the second-skin block produced by manipulating a flattened mesh, the methodology for creating this block excels in ease-of-automation, computational efficiency and compatibility with known pattern shaping practices. Therefore, it could easily be integrated with established apparel manufacturing practices.

For a first test in the digital environment, this study is considered a success. The fitting discrepancies noted in Figure 7 and Figure 8 are considered directly related to measurement errors which could be overcome in the digital environment. Since the highbust landmark is located according to highest armscye (axilla point), this girth dimension is best suited for a digital environment but placement of the axilla points proved problematic. In a digital environment, the landmark can remain horizontal through lower armscye areas where by hand any landmark must go around fleshy areas. Viewing the garment made from the digital measurements it became clear that further tilted planes were required to map this area.

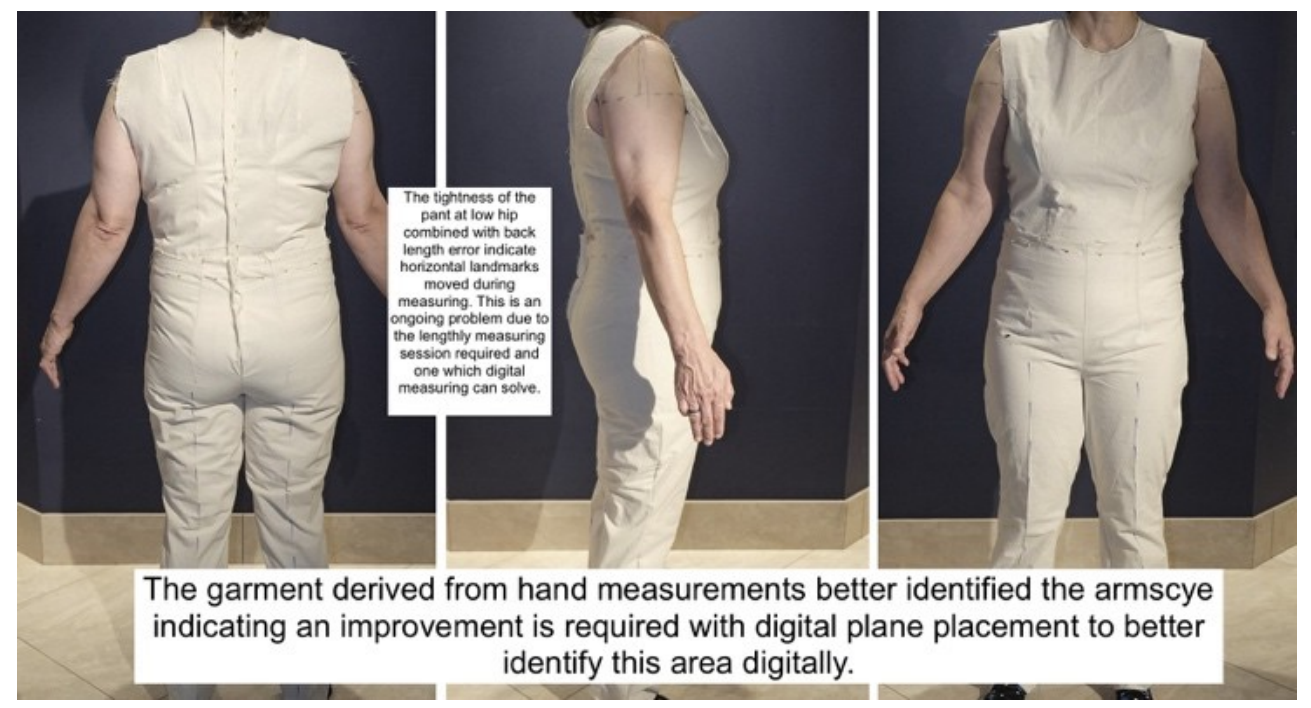

Figure $7 \sim$ Garment block from digital measures.

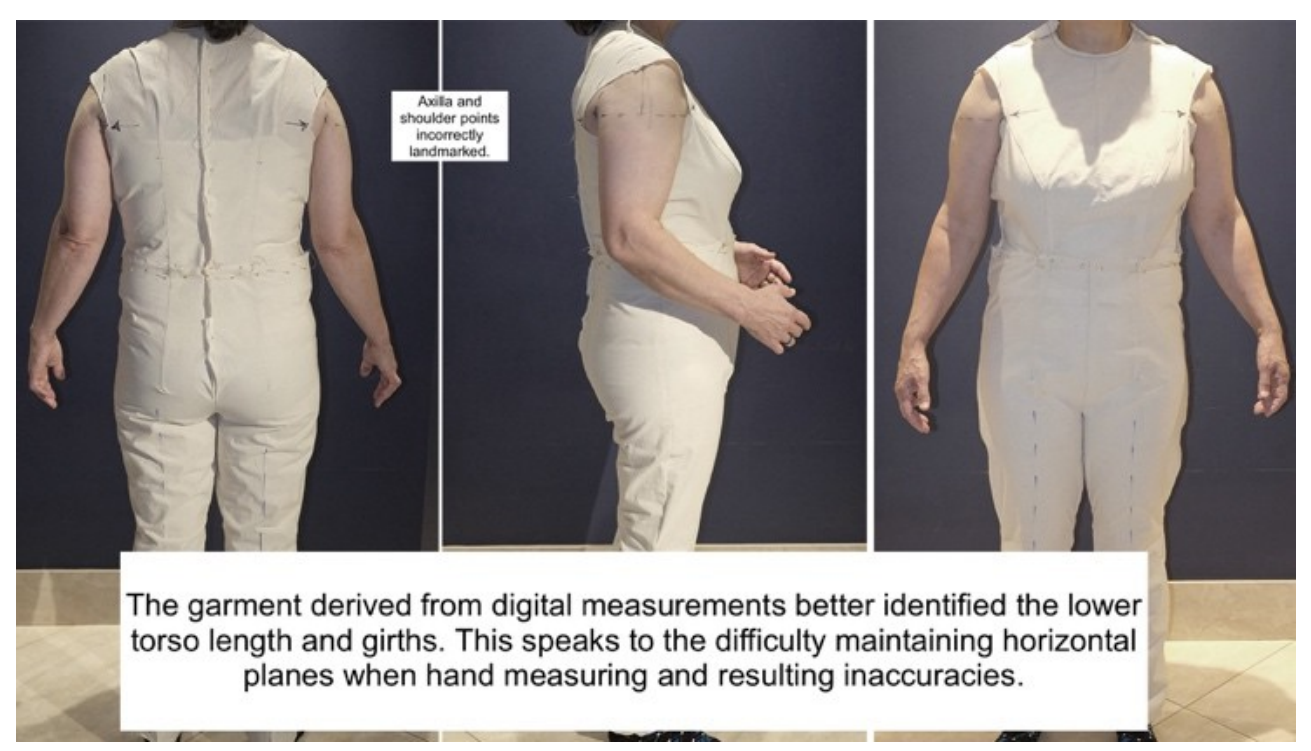

Figure $8 \sim$ Garment block from hand measures. 


\section{Discussion}

The Clone Block $®$ methodology has been extensively trialed in a bespoke environment and found to be effective for garment fit, despite continued difficulty with measurement data acquisition on a living breathing subject. As illustrated in Figure 4, the placement of strings and/or direct marks on the body is helpful but the process is still labour intensive and impractical in a business environment. Therefore, both time constraints and the ability of a subject to maintain patience for the duration of the measurement session have proven problematic and occasionally resulted in measurement error as noted in this study. Still, and it is interesting to note, despite measurement errors of up to $2 \mathrm{~cm}$, the Clone Block $®$ method of pattern-engineering still exceled in ability to replicate body morphology and proved exceptional for decreasing the need for sampling in a bespoke manufacturing environment. This success can be attributed to measurements missing from established pattern practice. The division of body girths has long been contemplated as a method for assessing body shape but traditional practice is inadequate to direct girth division. The few existing pattern-drafting texts recommend the use of halfbody measurements offer little guidance on dividing the body for measurement. Neither do the texts inform as to how the percentage of division should affect pattern-drafting for body shape. [16], [17] It has been suggested that automated virtual garment design can only be useful to fashion designers when the 3D and 2D CAD environments are driven by artificial neural networks mimicking the nonlinear thinking of humans. [18] For this to be realized, the iterative nature of pattern-drafting and the myriad of heuristic practices umbrellaed under the subjective garment fit label must be thoroughly and mathematically understood. This requires a Boolean logic perspective surrounding the non-linear thinking of garment fit and, most critically, this must be built upon a foundational, mathematical account of the body. In contrast with traditional pattern-drafting theory, that considers pattern shape

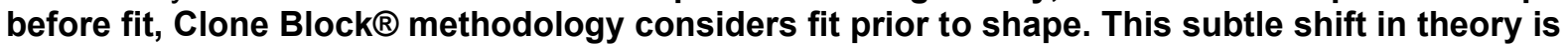
significant for several reasons critical to advancing apparel digitization.

\subsection{Improved Garment Sizing and Quantified Garment fit}

The traditional practice of assessing 1D girth dimensions (size chart body dimensions) for fit assessment essentially compares the body dimensions of a given individual against the dimensions of a fit model. This type of assessment offers little relevancy and at best, merely an estimation regarding garment fit, as it does not consider circumferential distributions critical for understanding body shape. Understanding the body from a 2D perspective permits a comparison of the body against garment dimensions for a precise understanding of ease.

As illustrated in Figure 9, analysis of garment fit relates to the data on a 2D pattern more than it relates to $1 \mathrm{D}$ body dimensions. The same analysis is not so easily accomplished on a pattern modelled as a polygon mesh, because these shapes do not relate to the weft and warp threads that define fabric grain against which garment fit and fabric drape are assessed. Only with foundational theories that regard the importance of the 2D developable garment pattern, can the added value of the 3D polygon mesh perspective be truly utilized for real-world garments.

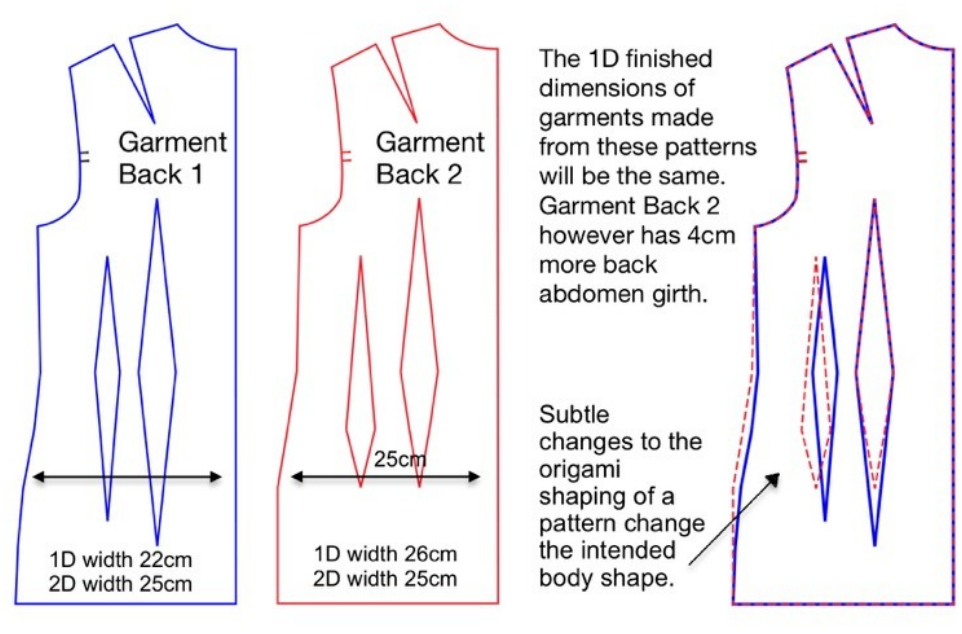
Mathematically quantifying the nuances of body shape is essential for the $3 \mathrm{D}$ to $2 \mathrm{D}$ interface. Without this the interaction of fabric on body can only be estimated.

Figure $9 \sim$ Ease and shaping distribution. 
Traditional practices for garment fit assessment either compare 1D (tape measure) measurements against a size chart or against garment dimensions. As discussed, this thinking is flawed because body shape is not revealed in 1D measurements making any assessment from such at best merely an estimation. Only with body shape assessed from an origami perspective, where the $3 \mathrm{D}$ is rendered as a $2 \mathrm{D}$ relational outline, can garment fit be quantified suitable for technological applications. While such assessment can be complex, technology holds the potential to simplify the process with automated measurement extraction. As discussed in Figure 2, only 2D measurements can identify the origami body shape differences between two individuals with similar dimensions. As previously illustrated in Figure 3 these differences are essential, to fully understand fit-preference with regard to visible and invisible ease distributions affecting fit, and ESSENTIAL for machine learning algorithms.

\subsection{Mass Garment Customization, MTM, and Body Shape Parameterization}

Nuances such as waist shaping, dart length, body width, breast allowance, shoulder shape, and posture allowance are but a few of the fitting concerns that have inhibited successful MTM fit due to a lack of pattern theory. Without a means by which to quantify the intricacies of body shape, MTM manufacturing can only accommodate width and length concerns related to the body, relying on the fit tolerance of fabric and ease to accommodate the other aspects of body shape. A direct relationship between body and pattern directs parameterization to fit any body regardless of shape or gender. As such, persistent difficulties associated with fitting outlier body shapes can be addressed. [19]

\subsection{Improved Design Process}

One of the most tumultuous workflows in the apparel manufacturing process is that which takes place between the designer and pattern-maker. 3D technologies are most impactful to workflow when a designer works along-side a pattern-maker. As designers are forced to consider new proportions suitable for body shape, body-to-pattern theory is essential for the understanding of ease distribution and how fabric falls over different body shapes.

Most CAD companies offer 3D-to-2D digital environments that automate virtual garment design. The exchange of data between the $3 \mathrm{D}$ and $2 \mathrm{D}$ environments permits the development of a garment pattern while the design process is finessed on a 3D avatar. Founded on traditional pattern-drafting theory, current workflows lack an accurate means by which to adjust darting, shaping, and ease distribution. Incorporating the landmarking and measuring used for the study herein, and using software such as Intelligent Shaping ${ }^{\circledR}$, enables a means by which pattern shaping and ease can be constantly monitored ensuring optimal ease distribution suitable for body shaping, while maintaining and directing garment fit throughout the design process. This 2D-to-3D interplay would take the guess-work out of patterndrafting for the designer who often has limited pattern-drafting expertise. [20]

With the Clone Block $\otimes$ inherently linked to measurements and optimal landmarking, the block and any garment designed from the block can immediately be updated from numerical data. This theory embedded in the 3D-2D interface automates the complexity of pattern-engineering for shape leaving both the designer and pattern-drafter to focus on design suitability. No longer having to rely purely on a sketch with questionable proportions, the 3D environment permits instant assessment of the design process. Most likely designers of the future will learn 3D skills in school and will be well-acquainted with automated 3D workflow. This workflow holds the potential to not only reduce sampling time, but also train designers to be more cognizant of the relationship between artistic expression and finished garment design.

\subsection{Required Theory Changes Affecting Established Manufacturing Processes}

Tailors, dressmakers, seamstresses and pattern drafters have relied heavily on pattern shaping experience and mentoring to compensate for a lack of theory. The slope of a center pant seam, breast dart size, waist shaping, dart length, leg balance, waistband slope, and body weight distribution are but a few of the self-learned "tricks of the trade" apparel professionals heuristically acquire to compensate for a lack of pattern theory. One needs not ponder how steep the learning curve will be when apparel professionals are provided a mathematical foundation for previously heuristic tasks but instead contemplate how quickly a trade may be radicalized when provided a mathematical foundation from which to build upon. In fact, automation itself may be just the catalyst to help practitioners gain a better understanding of how to exploit technology. Once practitioners can see how different body shapes drive pattern requirements, they will better appreciate how to implement fit requirements. 


\subsubsection{Changes to Measurement Data}

Suggesting a ten-fold increase in measurement data is bold but, in light of continued difficulty with the body-to-pattern relationships and continued suggestion from apparel research that changes are required, review is warranted. This study, using only a fraction of the body data accessible from a point cloud (and admittedly obtained in an experimental manner) offers a means by which body morphology can be quantified, ease isolated, and ultimately garment fit improved. The ease with which this data acquisition could be automated, without requiring significant change to traditional pattern-drafting practice, and the quality of output possible more than justifies a re-assessment of traditional measuring practices. Historically, a sixty- to ninety- minute hand measurement session in the fast-paced world of fashion, would not have been feasible, but technology now makes this possible. It will, however, require a recognition from industry as to the importance of extracting new data for the required paradigm shift.

How to hide the complexity of this data within size charts while still reaping the benefits must also be considered. With most sizing charts based on bust/waist/hip, the easiest way to acknowledge the body-to-pattern relationship without data overload is through the use of body shape sizing charts. This would permit a sub-categorization of individuals with similar 1D circumference girths. By familiar terms, this would mean any given garment size could be further broken out to accommodate hourglass, triangle, inverted triangle, round, and rectangle, whereas previously all shapes would have been accommodated in a single size. While extensive work has been done in this regard, the theory linking this extensive data to the $2 \mathrm{D}$ pattern has been missing. [20] Clone Block $\circledast$ theory, driven by underutilized scan data, can quantify body shape as it directly relates to the $2 \mathrm{D}$ pattern. As illustrated in Figure 10 no longer is size selection based on the incorrect use of 1D body dimensions. This permits an "apples to apples" comparison of the origami shaping inherent in all patterns for critical assessment of garment fit.
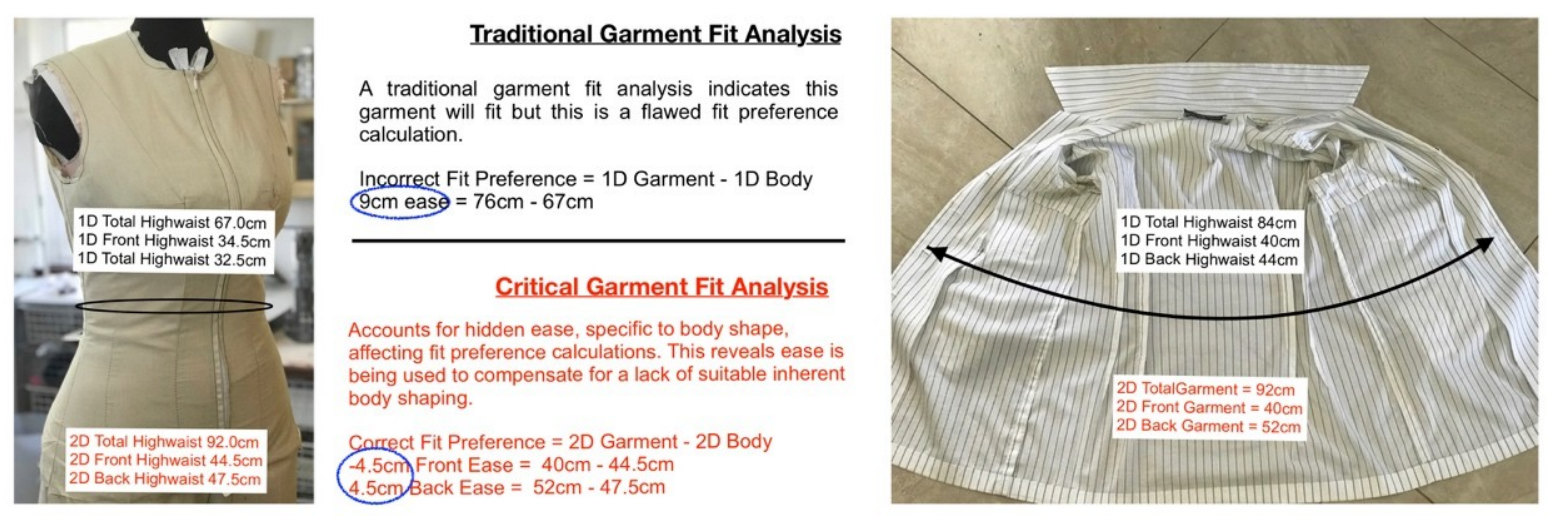

Figure 10 Critical garment fit assessment.

\subsubsection{Changes to Pattern-drafting Theory}

A direct relationship between body and pattern is only possible when patterns are landmarked to corresponding body landmarks. Such a change is substantial, but it does not need to happen overnight. Initially, not all patterns will be landmarked suitable for digitized technologies. MTM patterns will adopt the new landmarking first and Ready to Wear (RTW) patterns can be updated as size charts are updated for body shape. Further, much of this updating could be scripted for automation. Most importantly, the pattern-makers' workflow is not substantially changed. Pattern theory remains unchanged, with the exception that shaping for body morphology is now quantified for inclusion in the design process.

\subsection{Further Study}

While digitization of any industry is frequently found to be in conflict with the nuances of human interaction, fondly referred to as art, this has proven particularly difficult within apparel because the artistic expression of the garment designer must be practically put to pattern and reconciled with diverse consumer aesthetics, while complicated by the need for theory surrounding the heuristic aspects of garment fit. The Clone Block ${ }^{\circledR}$ method offers foundational theory upon which further theories addressing the art of design may be built. 


\subsubsection{Body Shape Size Charts}

With a mathematical foundation for garment fit, the apparel industry will benefit from a fresh perspective on body shape. A Clone Block ${ }^{\circledR}$ reassessment of body scanned libraries would provide data to differentiate individuals with similar 1D body measurements. Such data could be used to drive body shape sizing charts addressing the diverse needs of global demographics. This translates to more size charts addressing body shape, improved size prediction algorithms, and the ability to extend the demographic reach of sizing charts suitable for global demographics and finally inclusive of outlier body shapes. All of which directly address known industry concerns of customer dissatisfaction, unsold inventory and unsustainability.

\subsubsection{Ease Theory}

3D apparel CAD differs from 3D CAD for mechanical engineers, because the human body continually changes shape with movement. Shifts in posture, slight changes in leg stance, the act of breathing, digestion and even hormonal changes have subtle to profound changes on body shape and are the reasons traditional pattern-drafting is found to be inclusive of ease. While these have proven difficult to capture with simple physically measurements, they are not impossible to account for by designing for ease. 3D and 4D technologies are the optimal tools for numerically tracking changes to morphology. In particular, capturing the ease required for organic changes in body shape is of critical importance to functional apparel but equally impactful for apparel comfort. Further study should explore how organic movements alter the body-to-pattern relationship and use such data to develop a theory that accounts for ease.

\section{Conclusion}

Over a decade ago, Bye et al warned "... fit will depend upon our ability to combine the knowledge and skills of the $19^{\text {th }}$ century tailor or dressmaker with the strengths of manufacturing and information technology. The continued development and conservation of both our technological and intellectual resources is critical." [21] This speaks to the "chicken or egg scenario" in which apparel has been caught. The apparel industry could not develop a scan-to-pattern methodology without technology but technology could only advance so far without that methodology.

Reconciling artistic expression of the garment designer, a diverse consumer aesthetic, the practicalities of pattern-drafting for unique body shapes, and the requirements of digitization technologies has threatened to change both the art of design and pattern-drafting. Apparel digitization, however, has a built-in advantage. The 2D pattern, from which all garments arise, offers a bridge between the art and science required for apparel digitization. It will become essential for the artistic expression of the tailor, dressmaker, and fashion designer. The Clone Block $®$ measuring protocols, while they do require a paradigm shift with regard to measuring human form, provide the missing theory to bridge the voids between 3D and 2D technologies and propel apparel digitization forward.

\section{References}

[1] S. GILL, Y. WANG, M. AHMED, S. G. HAYES, A. R. G. HARWOOD, and J. GILL, "Scan to Pattern: How Body Scanning Can Help Transform Traditional Methods of Creating Pattern Blocks," 2018, pp. 236-240.

[2] E. Papahristou and N. Bilalis, "Can 3D Virtual Prototype Conquer the Apparel Industry?," Journal of Fashion Technology \& Textile Engineering, vol. 4, no. 2, Jul. 2016.

[3] P. Decaudin, D. Julius, J. Wither, L. Boissieux, A. Sheffer, and M. P. Cani, "Virtual garments: A fully geometric approach for clothing design," Computer Graphics Forum, vol. 25, no. 3, pp. 625634, 2006.

[4] A. Jung, S. Hahmann, D. Rohmer, A. Begault, L. Boissieux, and M. P. Cani, "Sketching folds: Developable surfaces from non-planar silhouettes," ACM Transactions on Graphics, vol. 34, no. 5, 2015.

[5] "3D Printed Fashion - The State of the Art in 2019 | All3DP." [Online]. Available: https://all3dp.com/2/3d-printed-fashion-the-state-of-the-art-in-2019/. [Accessed: 16-Sep-2019].

[6] P. Decaudin, D. Julius, J. Wither, L. Boissieux, A. Sheffer, and M. P. Cani, "Virtual garments: A fully geometric approach for clothing design," Computer Graphics Forum, vol. 25, no. 3, pp. 625634, 2006. 
[7] E. SCOTT and A. S. M. SAYEM, "Landmarking and Measuring for Critical Body Shape Analysis Targeting Garment Fit," in Proceedings of 3DBODY.TECH 2018 - 9th International Conference and Exhibition on 3D Body Scanning and Processing Technologies, Lugano, Switzerland, 16-17 Oct. 2018, 2018, pp. 222-235.

[8] V. E. Kuzmichev, N. A. Saharova, and G. I. Chistoborodov, "3D Body Scanning Technology for Virtual Design of System Body-Clothes," no. October, pp. 66-75, 2011.

[9] X. Peng and V. E. Kuzmichev, "Virtual method of predicting the accuracy of pattern blocks," IOP Conference Series: Materials Science and Engineering, vol. 459, no. 1, p. 012084, Dec. 2018.

[10] E. McKinney, S. Gill, A. Dorie, and S. Roth, "Body-to-Pattern Relationships in Women's Trouser Drafting Methods: Implications for Apparel Mass Customization," Clothing and Textiles Research Journal, vol. 35, no. 1, pp. 16-32, 2017.

[11] S. Gill, S. Hayes, and C. J. Parker, "3D Body Scanning: Towards Shared Protocols for Data Collection- Addressing the needs of the body scanning community for ensuring comparable data collection," no. January, 2016.

[12] S. Gill, C. J. Parker, S. Hayes, K. Brownbridge, P. Wren, and A. Panchenko, "The True Height of the Waist: Explorations of Automated Body Scanner Waist Definitions of the TC2 scanner," no. February, pp. 55-65, 2014.

[13] S. Gill, "Improving garment fit and function through ease quantification," Journal of Fashion Marketing and Management, vol. 15, no. 2. pp. 228-241, 2011.

[14] M. JANUSZKIEWICZ, C. J. PARKER, S. G. HAYES, and S. GILL, "Online Virtual Fit is not yet Fit for Purpose: An Analysis of Fashion e-Commerce Interfaces," pp. 210-217, 2017.

[15] I. Dabolina, A. Vilumsone, and J. Dabolins, "Anthropometrical Measurements for ThreeDimensional Clothing Design," 2010, no. October, pp. 404-410.

[16] S. GILL, M. AHMED, C. J. PARKER, and S. G. HAYES, "Not All Body Scanning Measurements Are Valid: Perspectives from Pattern Practice," no. October, pp. 43-52, 2017.

[17] K. Brownbridge, S. Gill, and S. Ashdown, "Brownbridge, KM and Gill, S and Ashdown, S ( 2013 ) Effectiveness of 3D Scanning in Establishing Sideseam Placement for Pattern Design . In: 4th International Conference and Exhibition on 3D Body Scanning Technologies . Downloaded from : Publisher : H," 2013.

[18] T. Jin Kang and S. Min Kim, "Optimized garment pattern generation based on three-dimensional anthropometric measurement," International Journal of Clothing Science and Technology, vol. 12, no. 4, pp. 240-254, Oct. 2000.

[19] S. Krzywinski and J. Siegmund, "3D Product Development for Loose-Fitting Garments Based on Parametric Human Models," IOP Conference Series: Materials Science and Engineering, vol. 254, no. 15, p. 152006, Oct. 2017.

[20] S. Krzywinski and J. Siegmund, "3D Product Development for Loose-Fitting Garments Based on Parametric Human Models," IOP Conference Series: Materials Science and Engineering, vol. 254, p. 152006, Oct. 2017.

[21] E. Bye, K. L. LaBat, and M. R. DeLong, "Analysis of Body Measurement Systems for Apparel," Clothing and Textiles Research Journal, vol. 24, no. 2, pp. 66-79, Mar. 2006. 